\title{
Transcranial Ultrasound in Comparison to MRI in Evaluation of Hypoxic Ischemic Injury in Neonates
}

\author{
Abd El-Aziz Kamal Aun*, Hassan Ali Hassan*, Wafik Ibrahim Ali*, Maged Mohamed Ali Ataky * \\ *Department of Radiodiagnosis, Al-Azhar University \\ Corresponding author: Maged Mohamed Ali Ataky, Mobile: (020)01010384245,E-Mail:dr.maged_mohamed_ali@hotmail.com
}

\begin{abstract}
Background: Hypoxic-ischemic injury is the most common cause of encephalopathy in newborns. Objectives: The goal of our study was to demonstrate the role of transcranial ultrasound (TCUS) compared to MRI in assessment of neonatal encephalopathy.

Patients and methods: Consecutive thirty sex neonates with hypoxic-ischemic encephalopathy (HIE) were included. All cases had undergone MR imaging as well as transcranial ultrasound study of the brain. The study was conducted between May 2015 and September 2017. They were collected from the Neonatology Intensive Care Unit at Elsayed Galal and El-Demerdash University Hospitals.

Results: Our study found that stages I and II showed mild to moderate HIE presented with peripheral pattern and white matter injuries while stage III revealed severe HIE presented with central pattern of injury (BGT). In addition, our study reported that all patients with stage III of HIE showed severe central pattern on MRI.

Conclusion: MRI imaging is considered a sensitive technique in detecting different MR patterns of encephalopathy in newborns. MRI placed other imaging modalities in diagnostic assessment and early predictor of future development of neurological abnormalities in neonates with encephalopathy.
\end{abstract}

Keywords: HII, TCUS, MRI, WD, Neonates

\section{Introduction}

Neonatal encephalopathy continues to be an important cause of neonatal mortality and morbidity in the United States. Hypoxic-ischemic injury (HII), which is also known as hypoxic-ischemic encephalopathy, refers to the subset of neonatal encephalopathy that result from a hypoxic or ischemic event, often in the setting of perinatal asphyxia, which leads to hypoxemia and hypercapnia. Hypotension and the resulting decreased cerebral blood flow are thought to lead to acidosis, release of inflammatory mediators and excitatory neurotransmitters, and formation of free radicals. HII is estimated to affect one to four infants per 1000 live births, depending on the criteria used to define infant encephalopathy. Between 20\% and 50\% of newborn infants with HII expire during the newborn period. Among the survivors, as many as $25 \%$ exhibit permanent neurologic damage, which typically manifests as cerebral palsy, mental retardation, or seizures ${ }^{(1)}$.

Multiple modalities have been assessed for their sensitivity in depicting HII, including, Transcranial Ultrasonography (TCUS), Computed Tomography (CT) and Magnetic Resonance (MR) Imaging. Newer diagnostic techniques such as Diffusion-weighted MR imaging and MR spectroscopy of the available imaging modalities. MR imaging is the most sensitive and is widely available. The addition of advanced modalities, par- ticularly MR spectroscopy and diffusion-weighted (DW) imaging may provide further insight into HIE, the potential for possible therapeutic intervention and increase specificity ${ }^{(2)}$. However, normal imaging findings in term neonates are substantially different from those in preterm infants, term infants and older children. An understanding of the normal development and anatomy of neonates is essential to recognize pathologic processes ${ }^{(3)}$.

HII in preterm Infants, in the setting of mild to moderate hypotension, the most common location for injury to the premature brain is the periventricular white matter, with ischemic parenchyma manifesting as PVL. In the setting of severe hypotension, because the thalami, brainstem, and cerebellum in the immature brain have high metabolic activity, they are more susceptible to injury and the insult manifests variable T2 signal at MR imaging and restricted diffusion on DWI. Coexisting periventricular white matter injury and germinal matrix hemorrhage may be present ${ }^{(3)}$.

Among the routine pulse sequences, diffusionweighted (DW) imaging is useful in the early days following a hypoxic-ischemic event and often depicts restricted diffusion within the first 24 hours that gradually increases over the next several days. DW imaging findings become falsely negative approximately 1 week after the hypoxic or ischemic event, a phenomenon known as pseudonormalization. 
Abd El-Aziz Aun et al.

These changes are more dramatic with the basal ganglia-thalamus pattern of injury. Overall, DW imaging findings should be evaluated in conjunction with ADC maps ${ }^{(4)}$.

Proton MR Spectroscopy may yield additional information and have increased specificity, particularly in the first 24 hours of life, when conventional imaging modalities are falsely negative or underestimate the degree of injury. Lactate, a marker of anaerobic metabolism, has a characteristic doublet peak at $1.3 \mathrm{ppm}$. Elevated lactate-creatinine and lactate-choline ratios have also been shown to indicate poor neurologic outcomes. Lactate levels are highest at 5-6 days and then gradually diminish, but lactate-N-acetyl aspartate and lactate-choline ratios may remain elevated. Other metabolite alterations that have been described in infants with HII include elevated choline-creatinine, glutamine-glutamate, lactate-acetyl aspartate, and lactate-choline and decreased acetyl aspartate-creatinine and acetyl aspartate-choline ratios ${ }^{(5)}$.

\section{Aim of the Work}

The aim of this work is comparison between the transcranial ultrasonography and Magnetic Resonance Imaging in assessment of neonatal hypoxic ischemic cerebral injury including the term and preterm infants and correlation with the severity of the clinical condition.

\section{Patients and Methods}

\section{Study population:}

Consecutive thirty sex neonates with HIE were included. All cases had undergone MR imaging as well as transcranial ultrasound study of the brain.
The study was conducted between May 2015 and September 2017. They were collected from the Neonatology Intensive Care Unit at Elsayed Galal and El-Demerdash University Hospitals. Informed consent was taken from all parents before inclusion in the study. The study was approved by the Ethics Board of Al-Azhar University.

\section{Inclusion criteria}

The study population comprised of term and preterm newborns (32-41 weeks) who suffered perinatal asphyxia. Perinatal asphyxia was defined as the need for delivery room resuscitation and development of clinical manifestations suggestive of HIE. All neonates were presented at time of examination by variable clinical picture of neonatal encephalopathy as disturbed conscious level (DCL) and low Apgar scoring (5 min score of $0-3$ ). The degree of encephalopathy was graded as mild, moderate and severe based on the staging

According to system of Sarnat and Sarnat,, in our study, 12 cases out of 36 patients were mild (stage I), 19 cases were moderate (stage II) and 5 cases were severe (stage III) (Table 1). All neonates were subjected to full clinical, neurological assessment and Apgar scoring by a pediatrician consultant, full history taking of any perinatal insult, full laboratory investigations including blood glucose and bilirubin, umbilical cord arterial blood sampling.

\section{Exclusion criteria}

Newborns with CNS infections, sepsis, respiratory distress, metabolic disorders, and major congenital malformations were excluded from the study.

Table (1): Sarnat and Sarnat's 3 Clinical Stages of Perinatal HIE

\begin{tabular}{|c|c|c|c|}
\hline Grade & Stage I & Stage II & Stage III \\
\hline Levelof consciousness & Hyperalert & Lethargic & Stuporous \\
\hline $\begin{array}{l}\text { Neuromuscular Control: } \\
\text {-Muscle tone } \\
\text {-Posture }\end{array}$ & $\begin{array}{l}\text {-Normal } \\
\text {-Mild distal } \\
\text { flexion }\end{array}$ & $\begin{array}{l}\text {-Mild hypotonia } \\
\text {-Strong distal flexion }\end{array}$ & $\begin{array}{l}\text {-Flaccid } \\
\text {-Intermittent } \\
\text { decerebration }\end{array}$ \\
\hline $\begin{array}{l}\text { Complex reflexes: } \\
\text { - Suck } \\
\text { - Moro }\end{array}$ & $\begin{array}{l}\text {-Weak } \\
\text {-Strong; low } \\
\text { threshold }\end{array}$ & $\begin{array}{l}\text {-Weak or absent } \\
\text {-Weak; incomplete; high } \\
\text { threshold }\end{array}$ & $\begin{array}{l}\text {-Absent } \\
\text {-Absent }\end{array}$ \\
\hline Pupils & Mydriasis & Miosis & $\begin{array}{l}\text { Variable ;often unequal; poor } \\
\text { light reflex }\end{array}$ \\
\hline Heart rate & Tachycardia & Bradycardia & Variable \\
\hline Seizures & None & $\begin{array}{l}\text { Common; focal or } \\
\text { multifocal }\end{array}$ & $\begin{array}{l}\text { Uncommon(excluding } \\
\text { decerebration) }\end{array}$ \\
\hline $\begin{array}{l}\text { Electroencephalogram } \\
\text { Findings }\end{array}$ & $\begin{array}{l}\text { Normal } \\
\text { (awake) }\end{array}$ & $\begin{array}{l}\text { Early:low voltage } \\
\text { continuous delta. } \\
\text { Later: periodic pattern } \\
\text { (awake) } \\
\text { Seizures: focal 1-to } 1-\mathrm{Hz} \\
\text { spike-and-wave }\end{array}$ & $\begin{array}{l}\text { Early: periodic pattern with } \\
\text { Isopotential phases } \\
\text { Later:totally isopotential }\end{array}$ \\
\hline
\end{tabular}




\section{Transcranial Ultrasound study}

The examinations were held on GE machine (Philips HD7XE.Thirty sex patients underwent a transcranial sonography at NICU. No sedation was needed. Imaging was performed using a highfrequency phased array transducer $(5-8 \mathrm{MHz})$ with a small footprint probe. Multiple acoustic windows were used to visualize as much of the central and peripheral structures of the brain as possible by using the anterior and posterior fontanels, as well as views through the temporal, mastoid and occipital areas. The transducer frequency was set at $8.2-11 \mathrm{MHz}$ for detection of cortical and/or sub-cortical abnormalities. We independently evaluated deep gray-matter structures, including basal ganglia, thalami and the brainstem. The angle of the transducer was varied in an attempt to evaluate the periphery of the brain with particular attention to the subcortical white matter and the gray-white matter differentiation in both of cerebral hemispheres.

\section{Magnetic Resonance Imaging \& Spectroscopy techniques}

\section{Magnetic Resonance Imaging:}

They were done on a 1.5 tesla super conducting system (Phillips MRI machine) using a head coil. Throughout the examination, neonates were supervised by an experienced neonatal pediatrician.

The MRI was performed in all neonates. An attempt was made to image the patients as soon as they were stable enough to be transported safely to the MR scanner. Nevertheless, factors such as patient instability, constraints of the MR schedule and availability of a physician to transport and monitor the infant, caused delays in performing MR imaging.

\section{Pre-imaging preparation:}

- Sedation was performed with oral $10 \%$ chloral hydrate $(50-75 \mathrm{mg} / \mathrm{kg}$ ) under supervision of an experienced neonatal paediatrician for irritable neonates; otherwise, patients were not sedated.

- Resuscitation equipment, infusion pump and with small-bore laryngoscope and endotracheal tubes should be kept in readiness.

\section{MR Spectroscopy:}

In $1 \mathrm{H}-\mathrm{MR}$ spectroscopy, proton spectra were acquired in 2 voxels, one located in the deep gray matter nuclei, including the thalamus and lentiform nucleus, and the other in the frontal or occipital intervascular boundary zone ("watershed zone") as well as at the site of the abnormality detected by conventional MRI and DW-MRI. The spectra were all acquired in conjunction with an MR imaging study of the brain included 4-mm sagittal T2 spinecho (SE) images, 4-mm axial T1, T2 SE images, and 4-mm coronal T2 SE images through the entire brain.

These images were used to guide the MRS volume selection, which was performed using single voxel, point-resolved spectroscopy (PRESS) technique. The size of the volume was dependent on the size of the basal ganglia, and area of interest in the frontal interboundary Zone but was mostly $20 \mathrm{x}$ $20 \times 20 \mathrm{~mm} 3$ (antero-posterior, left-to-right, and feetto-head directions). Contact with the periventricular white matter and the lateral ventricle was avoided as much as possible.

The spectra for each location was acquired with a TR of 2 seconds, a TE of 288 or (long), and 136(intermediate) milliseconds, and a total of 128 acquisitions. The MRS timings and voxel localization were chosen to maximize the detection of Lactate and to minimize the spectral contamination from extra cranial adipose tissues. If there was a need for a smaller voxel size, the number of acquisitions was increased to 192 or more. Localized shimming, phase correction, water suppression calibration and scan acquisition to eliminate artifacts caused by eddy currents were performed prior to acquisition of the spectra.

The abnormal MRS findings were depicted according to site of voxel at the region of interest (anterior water shed zone) and the basal ganglia region including rise of Lactate peak, appearing as doublet peak at $1.3 \mathrm{ppm}$ on spectra obtained with a short echo time $(35 \mathrm{msec})$, with inversion below the baseline on spectra obtained with intermediate echo times (135-144 msec). This inversion allows the lactate peak to be distinguished from the adjacent lipid peak (at 0.9-1.3 ppm), which does not invert at intermediate echo times.

\section{Interpretation of MRI findings:}

- Assessment of the ventricular system (size, intraventricular hemorrhage, germinal matrix hemorrhage).

- Reporting intra-or extra-axial hemorrhage

- Assessment of the white matter for signal changes.

- Assessment of the basal ganglia signal changes

- Monitoring of perivtricular leukomalcia (PVL).

\section{Statistical analysis}

Data analysis was performed using the SPSS statistical package, version 16.0 for windows. Statistical measures were expressed as means and 
Abd El-Aziz Aun et al.

standard deviation (SD). Predictive values of Cranial US findings for MRI findings were calculated and analyzed with $\chi 2$ tests and t-tests. In addition, sensitivity and specificity were calculated. $\mathrm{P}$ values less than 0.05 were considered statistically significant. Cross tables, statistics were used to assess the correlation between Cranial US and MRI findings and to evaluate the performance of both methods.

\section{Test sensitivity and specificity}

Test sensitivity is defined as the conditional probability that the test was positive if the condition is present. While test specificity is defined as conditional probability that the test was negative if the condition is absent.

\section{RESULTS}

The population enrolled in this study comprised of 36 neonates, $25(69.4 \%)$ males and $11(30.6 \%)$ females .The range of their gestational age was 31-41 weeks.

HIE neonates consisted of $27(75 \%)$ term cases their gestational age ranged from $37-41$ weeks at time of delivery while $9(25 \%)$ were preterm cases their gestational age ranged from 31-36 weeks at time of delivery.

Once clinical examination was suspected, TCUS and MRI studies were done within the first week after birth. TCUS and MRI examinations were performed on the same day in all of the studied populations.

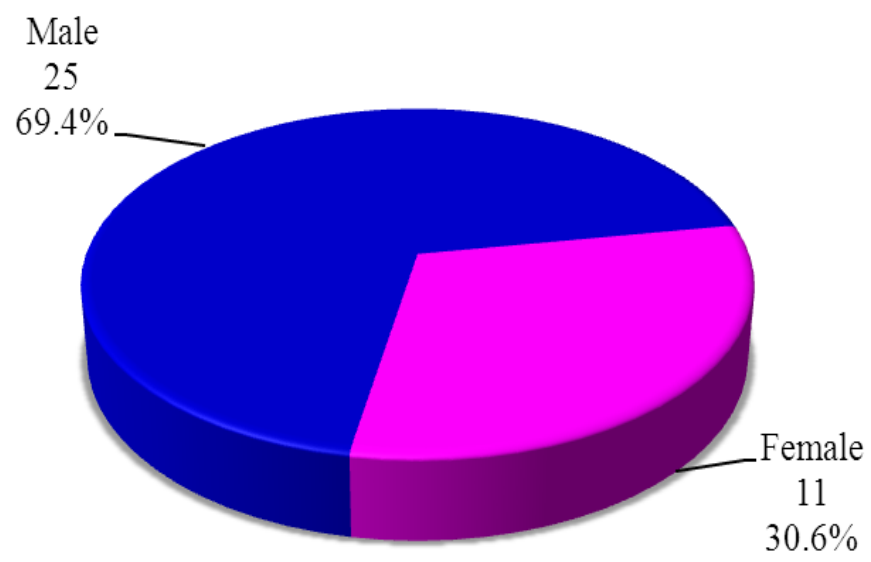

Figure (1): Pie Chart showing sex distribution

On their initial clinical staging for HIE (according to Sarnat System Staging), 12 (33.3\%) of cases were found to have stage I, while $19(52.8 \%)$ of patients were stage II and $5(13.9 \%)$ patients only were stage III (table 2).

\section{Table (2): Clinical staging of HIE (Sarnat System Staging)}

\begin{tabular}{|c|c|c|}
\hline Stage & N. & \% \\
\hline I & 12 & 33.3 \\
\hline II & 19 & 52.8 \\
\hline III & 5 & 13.9 \\
\hline
\end{tabular}

Correlation between the TCUS and MRI findings in evaluation of HIE:

In comparison with MRI, the number of positive and negative cases by TCUS was determined and thus the overall sensitivity, specificity and overall diagnostic accuracy were calculated. Our study included 36 neonates; MRI exams were positive in 30 cases and negative in 6 cases. TCUS was positive in 26 out of 36 neonates and negative in the remaining 10 cases. Compared to MRI, 24 cases of TCUS exams were true positive while 2 cases were false positive and 4 cases were true negatives while 6 cases were false negative. Among 26 patients identified having abnormal findings on TCUS, 24 cases were true positive had HIE findings on both TCUS and conventional MRI as follows; 13 cases elicited central basal ganglia injury, 10 cases elicited white matter injury and one case of germinal matrix hemorrhage grade I. Two cases were false positive, one case of white matter injury showed abnormal periventricular white matter hyper echogenicity on TCUS. The other case of central pattern of injury showed abnormal echogenicity of bilateral basal ganglia on TCUS. However, both cases appeared normal on conventional MRI.

Six cases were false negative diagnosed normal on TCUS showed abnormal findings on conventional MRI as follows; four cases elicited white matter injury pattern and one case elicited central basal ganglia injury pattern while one case elicited mixed pattern of both central and whiter matter patterns of injury. The overall sensitivity and specificity of TCUS in detecting imaging findings in 36 neonates with HIE compared to MRI were $80 \%$ and $66.6 \%$ respectively, yielding the overall diagnostic accuracy of TCUS compared to MRI was $78.9 \%$. The positive predictive value was $92.31 \%$, while the negative predictive value was $40 \%$ as shown in table (3). 
Transcranial Ultrasound in Comparison to MRI in Evaluation of Hypoxic Ischemic Injury in Neonates

Table (3): Correlation between the TCUS and MRI findings

\begin{tabular}{|c|c|c|c|c|c|c|c|c|}
\hline \multirow{3}{*}{ TCUS } & \multicolumn{6}{|c|}{ MRI } & \multirow{2}{*}{\multicolumn{2}{|c|}{ Chi-Square }} \\
\hline & \multicolumn{2}{|c|}{ Positive } & \multicolumn{2}{|c|}{ Negative } & \multicolumn{2}{|c|}{ Total } & & \\
\hline & $\mathbf{N}$ & $\%$ & $\mathbf{N}$ & $\%$ & $\mathbf{N}$ & $\%$ & $\chi^{2}$ & P-value \\
\hline Positive & 24 & 80.0 & 2 & 33.3 & 26 & 72.2 & \multirow{3}{*}{$5.428^{*}$} & \multirow{3}{*}{${ }^{\mathrm{FE}} \mathbf{p}=0.039^{*}$} \\
\hline Negative & 6 & 20.0 & 4 & 66.7 & 10 & 27.8 & & \\
\hline \multirow[t]{3}{*}{ Total } & 30 & 100.0 & 6 & 100.0 & 36 & 100.0 & & \\
\hline & \multicolumn{2}{|c|}{\begin{tabular}{l|l} 
Sensitivity \\
\end{tabular}} & Specificity & \multicolumn{2}{|c|}{ PPV } & NPV & \multicolumn{2}{|c|}{ Accuracy } \\
\hline & \multicolumn{2}{|c|}{80.0} & 66.67 & \multicolumn{2}{|c|}{92.31} & 40.0 & \multicolumn{2}{|c|}{77.78} \\
\hline
\end{tabular}

$\chi^{2}$ : Chi square test, FE: Fisher Exact *: Statistically significant at $\mathrm{p} \leq 0.05$

Correlation between the TCUS and MRI Diffusion findings in evaluation of HIE:

MRI diffusion exams were positive in 31 cases and negative in 5 cases. TCUS was positive in 26 out of 36 neonates and negative in the remaining 10 cases. Compared to MRI diffusion , 25 TCUS exams were true positive while 1case was false positive, and 4 cases were true negative while 6 cases were false negative. Among 26 cases identified having abnormal findings on TCUS, 25 cases were true positive had abnormal findings on TCUS and restricted diffusion as follows; 13 cases elicited central basal ganglia injury, 11 cases elicited white matter injury and one case of germinal matrix hemorrhage grade I had showed restricted diffusion in the periventricular white matter. The only false positive case identified was diagnosed clinically as HIE and TCUS and conventional MRI showed suspected deep gray matter abnormalities with associated hydrocephalus but no abnormality detected on diffusion MRI. Six cases were false negative diagnosed normal on TCUS showed restricted diffusion on MRI diffusion as follows; four cases of white matter injury pattern, one case of central basal ganglia injury pattern and one case of mixed pattern of both central and whiter matter injuries. Therefore, our study showed that DWI was useful in detection of mild to moderate HIE pattern of white matter injuries while TCUS was negative. The overall sensitivity and specificity of TCUS in detecting imaging findings in our 36 neonates with NE compared to MRI diffusion were $80.6 \%$ and 80 $\%$ respectively, yielding overall diagnostic accuracy of $80.5 \%$. The positive predictive value was $96.1 \%$, while the negative predictive value was $40 \%$ as shown in table (4).

Table (4): Correlation between the TCUS and MRI Diffusion findings

\begin{tabular}{|c|c|c|c|c|c|c|c|c|}
\hline \multirow{3}{*}{ TCUS } & \multicolumn{6}{|c|}{ MRI diff } & \multirow{2}{*}{\multicolumn{2}{|c|}{ Chi-Square }} \\
\hline & \multicolumn{2}{|c|}{ Positive } & \multicolumn{2}{|c|}{ Negative } & \multicolumn{2}{|c|}{ Total } & & \\
\hline & $\mathbf{N}$ & $\%$ & $\mathbf{N}$ & $\%$ & $\mathbf{N}$ & $\%$ & $\chi^{2}$ & P-value \\
\hline Positive & 25 & 80.6 & 1 & 20.0 & 26 & 72.2 & \multirow{3}{*}{$7.893^{*}$} & \multirow{3}{*}{${ }^{\mathrm{FE}} \mathbf{p}=0.015^{*}$} \\
\hline Negative & 6 & 19.4 & 4 & 80.0 & 10 & 27.8 & & \\
\hline \multirow[t]{3}{*}{ Total } & 31 & 100.0 & 5 & 100.0 & 36 & 100.0 & & \\
\hline & \multicolumn{2}{|c|}{ Sensitivity } & Specificity & \multicolumn{2}{|c|}{ PPV } & NPV & \multicolumn{2}{|c|}{ Accuracy } \\
\hline & \multicolumn{2}{|c|}{80.65} & 80.0 & \multicolumn{2}{|c|}{96.15} & 40.0 & \multicolumn{2}{|c|}{80.56} \\
\hline
\end{tabular}

$\chi^{2}$ : Chi square test, FE: Fisher Exact *: Statistically significant at $p \leq 0.05$

\section{Correlation between the TCUS and MRS findings in evaluation of HIE:}

MRS exams were positive in 32 cases and negative in 4 cases. TCUS was positive in 26 out of 36 neonates and negative in the remaining 10 cases (table 5). Compared to MRS Imaging, 26 TCUS exams were true positive while no false positive cases and 4 cases were true negatives while 6 cases were false negative. Among 26 cases identified having abnormal findings on TCUS, 26 cases were true positive had 
Abd El-Aziz Aun et al.

abnormal findings on both TCUS and MRS as follows; 13 cases elicited central basal ganglia injury and 11 cases elicited white matter injury. In addition to the case of germinal matrix hemorrhage grade I, the other case diagnosed clinically as HIE and showed suspected deep gray matter abnormalities with associated hydrocephalus on both TCUS and conventional MRI. The MRS imaging showed abnormal spectrum metabolite in both cases. Six cases were false negative diagnosed normal on TCUS showed abnormal metabolites within deep grey matter and white matter on MRS imaging as follows; four cases of white matter pattern injury, one case of central basal ganglia injury pattern and one case of mixed pattern of both central and whiter matter injuries. MRS findings of all above discussed cases showed abnormal metabolites within deep grey and white matters according to patterns of injury presenting with detectable lactate inverted double, decreased $\mathrm{N}$ acetyl aspartate and increased choline. Increased lactate/creatine and latate/choline ratios. Reduced $\mathrm{N}$ acetyl aspartate/creatine and $\mathrm{N}$ acetyl aspartate/choline ratios. The overall sensitivity and specificity of TCUS in detecting imaging findings in our 36 neonates with NE compared to MRS were $81.2 \%$ and $100 \%$ respectively, yielding overall diagnostic accuracy of $83.3 \%$. The positive predictive value was $100 \%$, while the negative predictive value was $40 \%$.

Table (5): Correlation between the TCUS and MRS findings

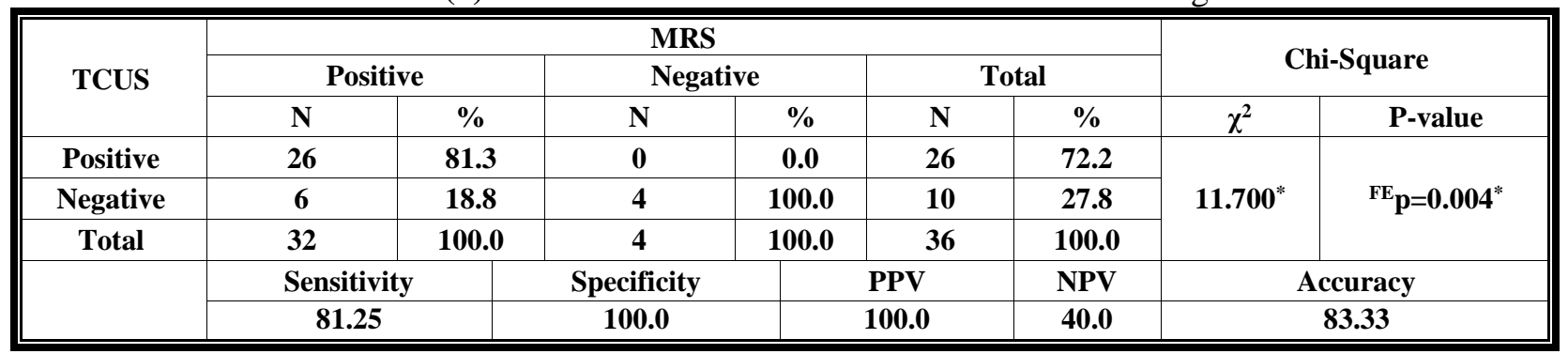

$\chi^{2}$ : Chi square test, FE: Fisher Exact *: Statistically significant at $\mathrm{p} \leq 0.05$

The previous results showed that MRS findings were more dependent on presence of disease than TCUS findings as proved by the higher Chisquare ratio, meaning the presence of a disease that probably lead to MRS findings than TCUS findings.

\section{Correlation between Conventional MRI and Diffusion DW-MRI findings}

MRI diffusion exams were positive in 31 cases and negative in 5 cases. MRI was positive in 30 out of 36 neonates and negative in the remaining 6 cases. Compared to MRI diffusion, 29 MRI exams were true positive while 1 case was false positive, and 4 cases were true negatives while 2 cases were false negative. Among 30 cases identified having abnormal findings on conventional MRI, 29 MRI cases were true positive had abnormal findings on conventional MRI and MRI diffusion as follows; 14 cases elicited central basal ganglia injury pattern, 10 cases elicited peripheral pattern of injury, one case elicited mixed pattern and 3 cases elicited white matter injury. In addition, one case of germinal matrix hemorrhage grade I showed restricted diffusion in the periventricular white matter on diffusion MRI. The only false positive case identified was the neonate with suspected grey matter abnormalities and associated hydrocephalus but had no abnormality on diffusion MRI. False negative two cases were diagnosed normal on conventional MRI showed restricted diffusion on MRI diffusion were of white matter injury pattern. The overall sensitivity and specificity of MRI in detecting imaging findings in our 36 neonates with NE compared to MRI diffusion were $93.5 \%$ and $80 \%$ respectively, yielding overall diagnostic accuracy of $91.6 \%$. The positive predictive value was $96.6 \%$, while the negative predictive value was $66.6 \%$ as shown in table (6).

Table (6): Correlation between CMRI and DW-MRI findings

\begin{tabular}{|c|c|c|c|c|c|c|c|c|}
\hline \multirow{3}{*}{ MRI } & \multicolumn{6}{|c|}{ MRI Diff } & \multirow{2}{*}{\multicolumn{2}{|c|}{ Chi-Square }} \\
\hline & \multicolumn{2}{|c|}{ Positive } & \multicolumn{2}{|c|}{ Negative } & \multicolumn{2}{|c|}{ Total } & & \\
\hline & $\mathbf{N}$ & $\%$ & $\mathbf{N}$ & $\%$ & $\mathbf{N}$ & $\%$ & $\chi^{2}$ & P-value \\
\hline Positive & 29 & 93.5 & 1 & 20.0 & 30 & 83.3 & \multirow{3}{*}{$16.769^{*}$} & \multirow{3}{*}{${ }^{F E} \mathbf{p}=0.001^{*}$} \\
\hline Negative & 2 & 6.5 & 4 & 80.0 & 6 & 16.7 & & \\
\hline \multirow[t]{3}{*}{ Total } & 31 & 100.0 & 5 & 100.0 & 36 & 100.0 & & \\
\hline & \multicolumn{2}{|c|}{\begin{tabular}{|l|l} 
Sensitivity & \\
\end{tabular}} & Specific & \multicolumn{2}{|c|}{ PPV } & NPV & \multicolumn{2}{|c|}{ Accuracy } \\
\hline & \multicolumn{2}{|c|}{93.55} & 80.0 & \multicolumn{2}{|c|}{96.67} & 66.67 & \multicolumn{2}{|c|}{91.67} \\
\hline
\end{tabular}

$\chi^{2}$ : Chi square test, FE: Fisher Exact *: Statistically significant at $\mathrm{p} \leq 0.05$ 
Transcranial Ultrasound in Comparison to MRI in Evaluation of Hypoxic Ischemic Injury in Neonates

\section{Correlation between conventional MRI and MRS findings}

MRS exams were positive in 32 cases and negative in 4 cases. MRI was positive in 30 out of 36 neonates and negative in the remaining 6 cases. Compared to MRS, 30 MRI exams were true positive while no false positive cases, and 4 cases were true negatives while 2 cases were false negative. Among 30 cases identified having abnormal findings on conventional MRI, 30 MRI cases were true positive had abnormal findings on conventional MRI and MRS as follows; 14 cases elicited central basal ganglia injury pattern, 10 cases elicited peripheral pattern, one case elicited mixed pattern and 3 cases elicited white matter injury. In addition to one case of germinal matrix hemorrhage grade I and the other case diagnosed clinically as HIE and showed suspected grey matter abnormalities with associated hydrocephalus. The MRS imaging showed abnormal spectrum metabolites in these two cases. False negative two cases were diagnosed normal on conventional MRI showed white matter abnormal metabolites on MRS imaging. MRS findings of all above discussed cases had showed abnormal metabolites within deep grey and white matters according to patterns of injury presenting with detectable lactate inverted double, decreased $\mathrm{N}$ acetyl aspartate and increased choline. Increased lactate/creatine and lactate/choline ratios. Reduced $\mathrm{N}$ acetyl aspartate/creatine and $\mathrm{N}$ acetyl aspartate/choline ratios. The overall sensitivity and specificity of MRI in detecting imaging findings in our 36 neonates with NE compared to MRS were $93.7 \%$ and $100 \%$ respectively, yielding overall diagnostic accuracy of $94.4 \%$. The positive predictive value was $100 \%$, while the negative predictive value was $66.6 \%$ as shown in table (7).

Table (7): Correlation between CMRI and MRS findings

\begin{tabular}{|c|c|c|c|c|c|c|c|c|}
\hline \multirow{3}{*}{ MRI } & \multicolumn{6}{|c|}{ MRS } & \multirow{2}{*}{\multicolumn{2}{|c|}{ Chi-Square }} \\
\hline & \multicolumn{2}{|c|}{ Positive } & \multicolumn{2}{|c|}{ Negative } & \multicolumn{2}{|c|}{ Total } & & \\
\hline & $\mathbf{N}$ & $29 \%$ & $\mathbf{N}$ & $\%$ & $\mathbf{N}$ & $\%$ & $\chi^{2}$ & $P$-value \\
\hline Positive & 30 & 93.8 & $\mathbf{0}$ & 0.0 & 30 & 83.3 & \multirow{3}{*}{$22.500^{*}$} & \multirow{3}{*}{${ }^{\mathrm{FE}} \mathbf{p}<0.001^{*}$} \\
\hline Negative & 2 & 6.3 & 4 & 100.0 & 6 & 16.7 & & \\
\hline \multirow[t]{3}{*}{ Total } & 32 & 100.0 & 4 & 100.0 & 36 & 100.0 & & \\
\hline & \multicolumn{2}{|c|}{ Sensitivity } & \multicolumn{2}{|c|}{ Specificity } & PPV & NPV & \multicolumn{2}{|c|}{ Accuracy } \\
\hline & \multicolumn{2}{|c|}{93.75} & \multicolumn{2}{|c|}{100.0} & 100.0 & 66.67 & \multicolumn{2}{|c|}{94.44} \\
\hline
\end{tabular}

$\chi^{2}$ : Chi square test, FE: Fisher Exact *: Statistically significant at $\mathrm{p} \leq 0.05$

The previous results showed that MRS findings were more dependent on presence of disease than MRI findings as proved by the higher Chi-square ratio, meaning that presence of a disease more probably would lead to MRS findings than MRI findings.

Correlation between the TCUS findings and the clinical staging of HIE

- Among the 12 patients with stage I HIE, 10 cases had normal TCUS and 2 patients had focal subcortical white matter abnormalities (water shed zones).

- Among the 19 patients with stage II HIE, 7 cases had normal TCUS findings and 12 had diffuse white matter abnormalities.

- Among the 5 patients with stage III HIE, the 5 patients had central pattern of affection with basal ganglia and thalamus abnormal hyperechogencity (table 8).

Table (8): Correlation between the TCUS findings and the clinical staging of HIE

\begin{tabular}{|c|c|c|c|c|c|c|}
\hline \multirow{2}{*}{$\frac{\text { STAGE }}{\text { TCUS }}$} & \multicolumn{2}{|c|}{1} & \multicolumn{2}{|c|}{2} & \multicolumn{2}{|c|}{3} \\
\hline & Count & $\%$ & Count & $\%$ & Count & $\%$ \\
\hline Normal & 10 & 83.3 & 7 & 36.8 & $\mathbf{0}$ & 0.0 \\
\hline Abnormal & 2 & 16.7 & 12 & 63.2 & 5 & 100.0 \\
\hline Total & 12 & 100 & 19 & 100 & 5 & 100 \\
\hline $\mathbf{X} 2$ & \multicolumn{6}{|c|}{$11.363^{*}$} \\
\hline MC(p value) & \multicolumn{6}{|c|}{$0.003 *$} \\
\hline
\end{tabular}

$\chi^{2}$ : Chi square test, MC: Monte Carlo *: Statistically significant at $\mathrm{p} \leq 0.05$ 
Therefore, our study found that TCUS could depict moderate HIE presented with white matter injuries and severe HIE presented with central pattern of injury (BGT) better than mild HIE presented with peripheral pattern of injury. In addition, our study reported that all patients with stage III of HIE showed severe central pattern on TCUS.

\section{Correlation between the MRI findings and the clinical staging of HIE}

- Among 12 patients with stage I HIE, 8 cases had normal MRI findings and 4 patients had water shed abnormality.

- Among the 19 patients with stage II HIE, 5 cases had normal MRI findings and 14 patients had white matter abnormalities.

- Among the 5 patients with stage III HIE, the 5 patients had severe central pattern of affection involving the basal ganglia and thalamus.

Table (9): Correlation between the MRI findings and the clinical staging of HIE.

\begin{tabular}{|c|c|c|c|c|c|c|}
\hline STAGE & 1 & & 2 & & 3 & \\
\hline MRI & Count & $\%$ & Count & $\%$ & Count & $\%$ \\
\hline Normal & 8 & 66.7 & 5 & 26.3 & $\mathbf{0}$ & 0.0 \\
\hline Abnormal & 4 & 33.3 & 14 & 73.7 & 5 & 100.0 \\
\hline Total & 12 & 100.0 & 19 & 100.0 & 5 & 100.0 \\
\hline $\mathbf{X} 2$ & \multicolumn{6}{|c|}{ 7.792* } \\
\hline MC(p value) & \multicolumn{6}{|c|}{$0.020 *$} \\
\hline
\end{tabular}

$\chi^{2}$ : Chi square test, MC: Monte Carlo *: Statistically significant at $\mathrm{p} \leq 0.05$

Our study found that stages I and II showed mild to moderate HIE presented with peripheral pattern and white matter injuries while stage III revealed severe HIE presented with central pattern of injury (BGT). Also, our study reported that all patients with stage III of HIE showed severe central pattern on MRI (table 9).

\section{Discussion}

Hypoxic ischemic encephalopathy (HIE) is a major cause of neurologic morbidity and mortality. It is the most common cause of neonatal encephalopathy that included in our study. HIE is characterized by diffuse white matter injury and neuronal disruption, leading to neurological disabilities such as cognitive impairment and cerebral palsy. Transcranial ultrasound is an operator dependant, bedside, easily available, cheap and quick method for imaging NE. MR imaging is an established tool to detect the timing, severity and extent of neonatal brain injury and it plays an important role in the prediction of neurological outcome ${ }^{(6)}$.

MR imaging is more sensitive than US and CT in detection of the HIE-related abnormalities, the presence of normal imaging does not rule out neurodevelopmental delay. Early MRI is mandatory as it can detect precisely the extent of brain injury ${ }^{(7)}$.

Hypoxia-ischemia-related brain injury can be seen more clearly with diffusion-weighted imaging
(DWI) than with conventional MRI, and abnormalities can be recognized earlier. DWI is, therefore, a useful clinical tool to assess the extent and pattern of injury. It proved to be excellent for detection of basal ganglia-thalamic as well as watershed injury ${ }^{(8)}$.

Proton magnetic resonance spectroscopy (1HMRS) plays an important role in the grading of the severity in $\mathrm{HIE}^{(9)}$.

The aim of our study was to investigate the value of TCUS versus MRI in detection of brain ischemic injuries in newborns with neonatal ischemic encephalopathy.

Consecutive thirty sex neonates with HIE were included in our study, their gestational age ranges from 32-41 weeks. All cases had undergone MR imaging as well as transcranial ultrasound study of the brain. The cases were classified in our study according to gestational age at time of delivery to full term 27 cases $(75 \%)$ and preterm 9 cases $(25 \%)$.

In agreement with our study, Ramachandran et al. ${ }^{(10)}$ in their study found that from total 50 patients. $82 \%$ were term babies and $18 \%$ were preterm babies.

In our study and the other studies mentioned before, we detected higher incidence of full term babies compared to preterm babies. This is explained by the usual high incidence of term deliveries compared to preterm deliveries. However, it is well 
known that the incidence of HIE is higher among the preterm babies compared to its incidence among full term babies due to incomplete brain maturation in preterm babies ${ }^{(11)}$.

In our study, TCUS was normal in 10 cases (27.8\%), BGT abnormality was detected in 14 cases $(38.9 \%)$ and WM abnormality was seen in 11 cases $(30.6 \%)$. One case $(2.7 \%)$ elicited germinal matrix haemorrhage grade I.

Genedi et al. (12) studied 38 neonates presented with HIE. TCUS was normal in 9 cases $23.7 \%$, central pattern injury seen in 15 cases $(39.5 \%)$ and white matter injury detected in 12 cases (31.6\%). Two cases $(5.2 \%)$ elicit germinal matrix haemorrhage. Therefore, our study TCUS findings were matching with Genedi $\boldsymbol{e}$ al. ${ }^{(12)}$ TCUS findings as regard pattern of injury.

Our study revealed that TCUS could depict central and white matter abnormalities better than peripheral lesions in patients with HIE.

So, our study findings are concomitant with Steggerda et al. ${ }^{(13)}$ who showed that TCUS has good sensitivity in detecting central (basal gangliathalamus) and white matter injuries in neonates with HIE rather than peripheral injuries.

In our study, conventional MRI was normal in six cases $(16.7 \%)$ while the remaining 30 cases (83.3\%) showed abnormalities as follows; central pattern injury detected in $41.6 \%$, white matter injury seen in $8.3 \%$, peripheral injury in $27.8 \%$. One case $2.8 \%$ elicited mixed pattern. In a study done by Genedi et al. ${ }^{(12)}$ that was conducted on 38 neonates with HIE. Conventional MRI was normal in 5 cases $(13.1 \%)$. The remaining 33 cases $(86.9 \%)$ showed abnormal findings as follows; central pattern injury seen in 16 cases $(42.5 \%)$ and white matter injury noted in 4 cases $(10.5 \%)$. One case $(2.6 \%)$ elicited mixed pattern and the other two cases (5.2\%) elicited germinal matrix haemorrhage.

Our study showed overall sensitivity and specificity of TCUS in detecting imaging findings in 36 neonates with HIE compared to MRI were $80 \%$ and $66.6 \%$ respectively, yielding the overall diagnostic accuracy of TCUS compared to MRI was $78.9 \%$. The positive predictive value was $92.31 \%$, while the negative predictive value was $40 \%$. Genedi et al. (12) found that the overall sensitivity and specificity of TCUS in detecting brain changes compared to MRI were $81.8 \%$ and $60 \%$ respectively, yielding overall diagnostic accuracy of $78.9 \%$. The positive predictive value was 93.1 while the negative predictive value was 33.3 (95\%). So, our study findings agreed with Genedi et al. ${ }^{(12)}$ findings as regards sensitivity, specificity, PPV, NPV and overall diagnostic accuracy. In addition, Herma et al. (14) agreed with our study where they found that transcranial ultrasound compared to MRI showed $82 \%$ of sensitivity in diagnosis of hypoxic-ischemic encephalopathy (HIE).

In our study there was statical difference detected between TCUS and MRI with higher efficacy of MRI in diagnosis of neonatal HIE (P value $=0.03)$. Babiker et al. ${ }^{(15)}$ reported the results of a study comparing MRI and TCUS in 150 neonates with suspected HIE. Transcranial US was normal in $50 \%$, increased periventricular echogenicity was seen in $32 \%$, germinal matrix haemorrhage was detected in $9 \%$ and basal ganglia (BG) abnormal echogenicity was noted in $18 \%$ of patients. Magnetic resonance imaging findings were normal in $29 \%$ of patients, basal ganglia (BG) abnormalities in $18 \%$ and germinal matrix haemorrhage in $10.6 \%$ of patients. In agreement with our study, Babiker et al. (15) found positive correlation between MRI and TCUS studies ( $P$ $=0.013$ ) with higher efficacy of MRI in evaluation of HIE.

Our study findings disagreed with Epelman $\boldsymbol{e t}$ al. ${ }^{(16)}$ findings as regard sensitivity, specificity, PPV, NPV and overall diagnostic accuracy. All of the described findings were much higher than recorded in our study.

In our study, MRI diffusion were positive in $86.1 \%$ and negative in $13.9 \%$. TCUS was positive in 26 out of 36 neonates and negative in the remaining 10 cases.

In our study, TCUS compared to DW-MRI showed that Six cases were false negative diagnosed normal on TCUS but showed restricted diffusion as follows; white matter pattern was seen in four cases, central pattern seen in one case and mixed pattern of injury was noted in one case. So, our study agreed with Genedi $\boldsymbol{e t} \boldsymbol{a l}{ }^{\left({ }^{(12)}\right.}$ as regards high efficacy of DWI in detection of mild to moderate HIE presented with white matter injuries while TCUS was negative.

In our study, MRS were positive in $88.9 \%$ and negative in $11.1 \%$. TCUS was regarded as positive in 26 out of 36 neonates and negative in the remaining 10 cases. Among 26 cases identified having abnormal findings on TCUS, 26 cases were true positive had abnormal findings on both TCUS and MRS.

Our study revealed abnormal metabolites spectrum at the sites of injury showing high lactate concentrations, inverted double lactate peak and 
Abd El-Aziz Aun et al.

increased $\mathrm{Cho} / \mathrm{Cr}$, $\mathrm{Lac} / \mathrm{Cr}$, and Lac/NAA peak ratios. On the other hand, it showed low $\mathrm{N}$-acetyl aspartate and reduced NAA/Cr and NAA/Cho peak ratios. Our results agreed with Epelman et al. ${ }^{(16)}$ where they found that from total 62 cases with HIE underwent TCUS and MRS scans, 33 cases had abnormal metabolites spectrum showing high lactate, inverted double lactate peak and low $\mathrm{N}$-acetyl aspartate as well as reduced $\mathrm{N}$ acetyl aspartate/creatine and $\mathrm{N}$ acetyl aspartate/choline ratios.

Our study MRI diffusion exams were positive in 31 cases and negative in 5 cases. MRI was positive in 30 neonates and negative in the remaining 6 cases. Conventional MRI compared to MRS revealed that 29 cases with HIE had abnormal findings on conventional MRI with restricted diffusion on DW-MRI. Therefore, our study findings matched with Jose et al. ${ }^{(17)}$ MRI and DW-MRI findings regarding central and peripheral pattern of injuries with corresponding restricted diffusion. Our study showed statistical difference between TCUS and DW-MR with higher efficacy of DWI in diagnosis of neonates with HIE (P value $=0.01)$. In agreement with our study Li $\boldsymbol{e t}$ al . (18) in their study found positive correlation between MRI and DW-MRI studies ( $\mathrm{P}=0.04)$ with higher efficacy of MRI in evaluation of HIE.

In our study, MRS were positive in 32 cases and negative in 4 cases. MRI was regarded as positive in 30 neonates and negative in the remaining 6 cases. Conventional MRI compared to MRS revealed that 30 cases with HIE had abnormal findings on both conventional MRI and MRS. Our study showed abnormal MRS findings with significant higher concentration of cerebral lactate and increased $\mathrm{Cho} / \mathrm{Cr}$ and $\mathrm{Lac} / \mathrm{Cr}$ peak ratios. On the other hand showing low $\mathrm{N}$-acetyl aspartate and reduced NAA/Cr. So, our study findings matched with Wu et al. ${ }^{(19)}$ where they reported that MRI and MRS findings showed abnormal metabolite spectrum findings at both central and watershed zones injuries.

Our study overall sensitivity and specificity of MRI in detecting imaging findings compared to $1 \mathrm{H}-$ MRS were $93.7 \%$ and $100 \%$ respectively, yielding overall diagnostic accuracy of $94.4 \%$. The positive predictive value was $100 \%$, while the negative predictive value was $66.6 \%$. So, our study findings disagreed with Mansour et al. (20) as regard sensitivity, specificity, PPV, NPV and overall diagnostic accuracy. All of the described findings were lower than recorded in our study. While agreed with our study as regards abnormal metabolites spectrum findings at the sites of affection showing decreased mean peak-ratios of the $\mathrm{NAA} / \mathrm{Cr}$, and NAA/Cho. On the other hand, they showed increased mean peak-ratios of the $\mathrm{Cho} / \mathrm{Cr}, \mathrm{Lac} / \mathrm{Cr}$, and Lac/NAA.

In our study, HIE was classified into mild, moderate and severe encephalopathy, respectively according to modified Sarnat and Sarnat classification. 12 cases were grade I, 19 cases grade II and 5 cases grade III.

Our study revealed correlation of MRI findings and HIE clinical staging as follows:

- Among 12 patients with stage I HIE, 8 cases had normal MRI findings and 4 patients had watershed abnormality.

- Among the 19 patients with stage II HIE, 5 cases had normal MRI findings and 14 patients had white matter abnormalities.

- Among the 5 patients with stage III HIE, the 5 patients had central pattern of affection involving the basal ganglia and thalamus.

Our results agreed with Dağ $\boldsymbol{e t}$ al. ${ }^{(21)}$ where they found that stages I and II showed mild to moderate HIE presented with peripheral pattern and white matter injuries while stage III revealed severe HIE presented with central pattern of injury (BGT). Also, agree with our study as regards good correlation between stage III HIE and MRI findings as all patients with stage III of HIE where they showed severe central pattern on MRI.

\section{Conclusion}

MRI is the most sensitive and specific modality for evaluating neonatal HIE because of the cost advantage, portability, and availability. Early conventional MRI and DWI are mandatory as they can detect precisely the extent of brain injury compared to TCUS. In addition to advanced MR imaging techniques, particularly MR spectroscopy can provide valuable diagnostic information especially when conventional MRI and DWI were negative or under estimate the degree of injury.

Performing MRI in these neonates need particular care regarding patients transport, monitoring of vital signs and use of appropriate coil and protocol for excellent image quality.

MRI T1- and T2- weighted imaging are most sensitive for suspected cases of HIE after 2-3 days. Diffusion-weighted imaging is sensitive for the detection of injury in the first 24 hours, during which time conventional T1- and T2-weighted images may appear normal. Abnormalities seen at diffusion weighted imaging generally peak at 3-5 days and subsequently "pseudonormalize" by about the end of the 1st week. MR spectroscopy is more sensitive to injury and more indicative of the severity of injury in the first 24 hours after a hypoxic-ischemic episode 
when conventional and diffusion-weighted MR imaging may yield false-negative findings or lead to significant underestimation of the extent of injury.

Therefore, knowing the time of onset of encephalopathy is important when we perform MRI especially DWI and MRS for better estimation of the extent of HIE injury.

Recommendations: We recommend that the ultimate radio-diagnostic protocol for cases of HIE is a combination of both TCUS and MR imaging.

\section{References}

1. Ghei SK, Zan E, Nathan JE et al. (2014): MR imaging of hypoxic-ischemic injury in term neonates: pearls and Pitfalls. Radiographics, 34: 1047-1061.

2. Chao CP, Zaleski CG, Patton AC (2010): Neonatal hypoxic-ischemic encephalopathy: multimodality imaging findings. Radiographics, 26: S159-S172.

3. Izbudak I, Grant PE (2011): MR imaging of the term and preterm neonate with diffuse brain injury. Magn. Reson. Imaging Clin. N. Am., 19 (4): 709-731.

4. Vermeulen RJ, van Schie PE, Hendrikx L et al. (2013): Diffusion-weighted and conventional MR imaging in neonatal hypoxic ischemia: two-year follow-up study. Radiology, 249 (2): 631-639.

5. Huppi PS, Lodygensky.GA, Barker PB et al. (2014): For the MRS consensus group 2. Clinical proton MR spectroscopy in central nervous system disorders. Radio. Graphics, 270: 750-791.

6. Huang ZH, Sun Y, Chen C (2011): Progress in encephalopathy of prematurity. Zhongguo. Dang. Dai. Er. Ke. Za. Zhi., 13: 771-775.

7. Malik GK, Pandey M, Kumar R et al. (2010): MR imaging and in vivo proton spectroscopy of the brain in neonates with hypoxic ischemic encephalopathy. Eur. J. Radiol., 43: 6-13.

8. Babiker MS, Omer AM, Oufi A (2016): Evaluation of neonatal hypoxic-ischemic encephalopathy by MRI and ultrasound. J. Diagnostic Med. Sonography, 29 (4): 159-164.

9. Chau V, Poskitt KJ, Miller SP (2012): Advanced neuroimaging techniques for the term newborn with encephalopathy. Pediatr. Neurol., 40: 181-188.

10. Ramachandran S, Sripathi S (2017): To evaluate the role of MRI in infants with suspected hypoxic ischemic encephalopathy and prognosticating neurological outcome at end of one year. International J. Res. Med.
Sci., 5 (5): 1893-1897.

11. Upadhyay $P$, Patil $K U$, Kuber $R$ et al. (2014): Evaluation of hypoxic-ischaemic events in preterm neonates using trans cranial ultrasound. International J. Healthcare Biomed Res., 3: 67-72.

12. Genedi EA, Usman NM, El-deeb MT (2016): Magnetic resonance imaging versus transcranial ultrasound in early identification of cerebral injuries in neonatal encephalopathy. Egypt J. Radio. Nuc. Med., 47: 297-304.

13. Steggerda SJ, Leijser LM, Walther FJ et al. (2009): Neonatal cranial ultrasonography: how to optimize its performance. Early Hum. Dev., 85: 93-99.

14. Herma P, Kalola J, Sood M et al. (2018): Transcranial ultrasound: efficient screening tool for detection of hypoxic brain injury in neonates - study of 50 patients. Int. J. Contemporary Med. Surg. Radiol., 3 (3): 9395.

15. Bednarek $\mathrm{N}$, Mathur $\mathrm{A}$, Inder $\mathrm{T}$ et al. (2012): Impact of therapeutic hypothermia on MRI diffusion changes in neonatal encephalopathy. Neurology, 78: 1420-1427.

16. Epelman M, Daneman A, Kellenberger CJ et al. (2010): Neonatal encephalopathy: a prospective comparison of head US and MRI. Pediatr. Radiol., 40: 1640-1650.

17. Jose O, Sheena V (2017): MRI changes of brain in newborns with hypoxic ischemic encephalopathy clinical stage II or stage III- a descriptive study. Int. J. Med. Pediatr. Oncol., 3 (1): 29-33.

18. Li AM, Chau V, Poskitt KJ et al. (2009): White matter injury in term newborns with neonatal encephalopathy. Pediatr. Res., 65: 85-89.

19. Wu TW, Tamrazi B, Hsu KH et al. (2018): Cerebral lactate concentration in neonatal hypoxic-ischemic encephalopathy: in relation to time, characteristic of injury, and serum lactate concentration. Front Neurol., 9: 293.

20. Mansour OY, Hanfy D, Fathy $S$ et al. (2017): Magnetic resonance imaging versus proton magnetic resonance spectroscopy in neonatal hypoxic ischemic encephalopathy in Egyptian population: pilot study. J. Neurol. Neurol. Sci. Disord., 3 (2): 43-50.

21. Da ̆ Y, Firat AK, Karakaş HM et al. (2009): Clinical outcomes of neonatal hypoxic ischemic encephalopathy evaluated with diffusionweighted magnetic resonance imaging. Diagn. Interv. Radiol., 12: 109-114. 